\title{
Two-Temperature Generalized Thermoelastic Infinite Medium with Cylindrical Cavity Subjected To Time Exponentially Decaying Laser Pulse
}

\author{
Eman A. N. Al-Lehaibi \\ Department Mathematic, College of Science and Arts Sharoura, Najran University, KSA
}

\begin{abstract}
(Received 14 March 2015; accepted 17 September 2015)
The present work is devoted to a study of the induced temperature and stress fields in an elastic infinite medium with cylindrical cavity under the purview of two-temperature thermoelasticity. The medium is considered to be an isotropic homogeneous thermoelastic material. The bounding plane surface of the cavity is loaded thermally by time exponentially decaying laser pulse. An exact solution of the problem is obtained in Laplace transform space, and the inversion of Laplace transforms have been carried numerically. The derived expressions are computed numerically for copper, and the results are presented in graphical form.
\end{abstract}

\section{INTRODUCTION}

In-depth research has been conducted on generalized thermoelasticity theories in solving thermoelastic problems in place of the classical uncoupled/coupled theory of thermoelasticity. The absence of any elasticity term in the heat conduction equation for uncoupled thermoelasticity appears to be unrealistic, since the produced strain causes variation in the temperature field due to the mechanical loading of an elastic body. The parabolic type of heat conduction equation results in an infinite velocity of thermal wave propagation, which also contradicts the actual physical phenomena. By introducing the strain-rate term in the uncoupled heat conduction equation, the analysis to incorporate coupled thermoelasticity has been extended by Biot. ${ }^{1}$ Although the first paradox was over, the parabolic type partial differential equation of heat conduction remains, which leads to the paradox of infinite velocity of the thermal wave. To eliminate this paradox, generalized thermoelasticity theory was developed subsequently. Due to the advancement of pulsed lasers, fast burst nuclear reactors, and particle accelerators, which can supply heat pulses with a very fast time-rise, Bargmann. $^{2}$ and Boley ${ }^{3}$ generalized thermoelasticity theory is receiving serious attention. Chandrasekharaiah reviewed the development of the second sound effect. ${ }^{4}$ Recently, mainly two different models of generalized thermoelasticity are being extensively used: one proposed by Lord and Shulman and the other proposed by Green and Lindsay. ${ }^{5,6}$ Lord and Shulman theory (L-S) suggests one relaxation time, and according to this theory, only Fourier's heat conduction equation is modified; however, Green and Lindsay theory (G-L) suggests two relaxation times, and both the energy equation and the equation of motion are modified.

The so-called ultra-short lasers are those with pulse duration ranging from nanoseconds to femtoseconds in general. In the case of ultra-short-pulsed laser heating, the high-intensity energy flux and ultra-short duration laser beam, have introduced situations where very large thermal gradients or an ultra-high heating speed may exist on the boundaries, according to Sun et al. ${ }^{7}$ In such cases, as pointed out by many investigators, the classical Fourier model, which leads to an infinite propagation speed of the thermal energy, is no longer valid for Tzou. ${ }^{8,9}$ The non-Fourier effect of heat conduction takes into account the effect of mean free time (thermal relaxation time) in the energy carrier's collision process, which can eliminate this contradiction. Wang and Xu have studied the stress wave induced by nanoseconds, picoseconds, and femtoseconds laser pulses in a semi-infinite solid. ${ }^{10}$ The solution takes into account the non-Fourier effect in heat conduction and the coupling effect between temperature and strain rate. It is known that characteristic elastic waveforms are generated when a pulsed laser irradiates a metal surface.

The two-temperatures theory of thermoelasticity was introduced by Gurtin and Williams, ${ }^{11}$ Chen and Gurtin, ${ }^{12}$ and Chen et al., ${ }^{13,14}$ in which the classical Clausius-Duhem inequality was replaced by another one depending on two temperatures; the conductive temperature $\varphi$ and the thermodynamic temperature $T$, the first is due to the thermal processes, and the second is due to the mechanical processes inherent between the particles and the layers of elastic material, this theory was also investigated by Iean. ${ }^{15}$ Abbas solved many problems that discussed the two-temperature theory of thermoelasticity and also the thermoelastic medium with cylindrical cavity. ${ }^{16-20}$

Only in the last decade has the theory of two-temperature thermoelasticity been noticed, developed in many works, and found its applications mainly in the problems in which the discontinuities of stresses have no physical interpretations. Among the authors who contribute to this theory, Quintanilla studied existence, structural stability, convergence, and spatial behavior for this theory. ${ }^{21}$ Youssef introduced the generalized Fourier law to the field equations of the two-temperature theory of thermoelasticity and proved the uniqueness of solution for homogeneous isotropic material. ${ }^{22,23}$ Puri and Jordan recently studied the propagation of harmonic plane waves ${ }^{23}$ and Magaa and Quintanilla ${ }^{24}$ have studied the uniqueness and growth solutions for the model proposed by Youssef. ${ }^{25}$

The present work is devoted to a study of the induced tem- 
perature and stress fields in an elastic infinite medium with cylindrical cavity under the purview of two-temperature thermoelasticity. The medium is considered to be an isotropic homogeneous thermoelastic material. The bounding plane surface of the cavity is loaded thermally by non-Gaussian laser beam with pulse duration of $2 \mathrm{ps}$. An exact solution of the problem is obtained in Laplace transform space, and the inversion of Laplace transforms have been carried numerically. The derived expressions are computed numerically for copper, and the results are presented in graphical form.

\section{THE GOVERNING EQUATIONS}

Consider a perfectly conducting elastic infinite body with cylindrical cavity occupies the region $R \leq r<\infty$ of an isotropic homogeneous medium whose state can be expressed in terms of the space variable $r$ and the time variable $t$ such that all of the field functions vanish at infinity.

We can use the cylindrical system of coordinates $(r, \psi, z)$ with the z-axis lying along the axis of the cylinder. Due to symmetry, the problem is one-dimensional with all the functions considered depending on the radial distance $r$ and the time $t$. It is assumed that there is no external forces act on the medium.

Thus the field equations in cylindrical one dimensional case can be put as in: ${ }^{25}$

$$
\begin{gathered}
(\lambda+2 \mu) \frac{\partial e}{\partial r}-\gamma \frac{\partial T}{\partial r}=\rho \frac{\partial^{2} u}{\partial t^{2}} \\
\nabla^{2} \varphi=\frac{\rho C_{E}}{K}\left(\frac{\partial}{\partial t}+\tau_{o} \frac{\partial^{2}}{\partial t^{2}}\right) \theta \\
+\frac{T_{o} \gamma}{K}\left(\frac{\partial}{\partial t}+\tau_{o} \frac{\partial^{2}}{\partial t^{2}}\right) e-\frac{\rho}{K}\left(1+\tau_{o} \frac{\partial}{\partial t}\right) Q \\
\varphi-T=a \nabla^{2} \varphi \\
\sigma_{r r}=2 \mu \frac{\partial u}{\partial r}+\lambda e-\gamma\left(T-T_{o}\right) \\
\sigma_{\psi \psi}=2 \mu \frac{u}{r}+\lambda e-\gamma\left(T-T_{o}\right) \\
\sigma_{z z}=\lambda e-\gamma\left(T-T_{o}\right) \\
\sigma_{z r}=\sigma_{\psi r}=\sigma_{z \psi}=0 \\
e=\frac{1}{r} \frac{\partial(r u)}{\partial r}
\end{gathered}
$$

where $\nabla^{2}=\frac{\partial^{2}}{\partial r^{2}}+\frac{1}{r} \frac{\partial}{\partial r}, \lambda, \mu$, Lames constants, $\rho$ density, $C_{E}$ specific heat at constant strain, $\alpha_{T}$ coefficient of linear thermal expansion, $\lambda=(3 \lambda+2 \mu) \alpha_{T}, t$ is the time, $T$ is the temperature, $T_{0}$ is the reference temperature, $\theta=\left(T-T_{o}\right)$ is the thermo-dynamical temperature increment such that $\frac{|\theta|}{T_{o}}<<1, \varphi$ is the heat conductive temperature, $\sigma_{i j}, i, j=r, \psi, z$ are the components of stress tensor, $e$ is the cubic dilatation, $u$ is the displacement, $K$ is the thermal conductivity, $\tau_{o}$ is the relaxation time, $a$ is non-negative parameter (two-temperature parameter), and $Q$ is the heat source per unit mass.

\subsection{The Mathematical Modeling}

The Fourier heat transfer equation due to time exponentially decaying laser pulse for a one dimensional body can be written as: ${ }^{7}$

$$
\begin{aligned}
\nabla^{2} \varphi=\frac{\rho C_{E}}{K}\left(\frac{\partial}{\partial t}+\right. & \left.\tau_{o} \frac{\partial^{2}}{\partial t^{2}}\right) \theta+\frac{\gamma T_{o}}{K}\left(\frac{\partial}{\partial t}+\tau_{o} \frac{\partial^{2}}{\partial t^{2}}\right) e \\
& -\frac{\rho I_{1} \delta}{K}\left(1+\tau_{o} \frac{\partial}{\partial t}\right)\left[e^{-v t-\delta r}\right] ;
\end{aligned}
$$

where $I_{1}=\left(1-r_{f}\right) I_{0}$ is the power intensity after surface reflection; $I_{0}$ is laser peak power intensity; $r_{f}$ is reflection coefficient; $v$ is laser pulse parameter; and $\delta$ is absorption coefficient.

\subsection{Dimensionless of the Governing Equations}

For convenience, we shall use the following nondimensional variables: ${ }^{25}$

$$
\begin{aligned}
\left(r^{\prime}, u^{\prime}, R^{\prime}, R_{0}\right) & =c_{o} \eta\left(r, u, R^{\prime}, R_{0}^{\prime}\right), \\
\left(t^{\prime}, \tau_{o}^{\prime}\right) & =c_{o}^{2} \eta\left(t, \tau_{o}\right), \\
\theta^{\prime} & =\frac{T-T_{o}}{T_{o}} \\
\varphi^{\prime} & =\frac{\varphi-T_{o}}{T_{o}} \\
\sigma^{\prime} & =\frac{\sigma}{\mu}
\end{aligned}
$$

where $c_{0}^{2}=\frac{\lambda+2 \mu}{\rho}$ and $\eta=\frac{\rho C_{E}}{K}$.

Hence, we obtain (where the primes are suppressed for simplicity)

$$
\nabla^{2} e-b \nabla^{2} \theta=\frac{\partial^{2} e}{\partial t^{2}}
$$

$$
\begin{gathered}
\nabla^{2} \varphi=\left(\frac{\partial}{\partial t}+\tau_{o} \frac{\partial^{2}}{\partial t^{2}}\right) \theta+ \\
\varepsilon_{1}\left(\frac{\partial}{\partial t}+\tau_{o} \frac{\partial^{2}}{\partial t^{2}}\right) e-\varepsilon_{2}\left(1+\tau_{o} \frac{\partial}{\partial t}\right)\left[e^{-v t-\delta r}\right] \\
\varphi-\theta=\omega \nabla^{2} \varphi \\
\sigma_{r r}=\beta^{2} \frac{\partial u}{\partial r}+\left(\beta^{2}-2\right) \frac{u}{r}-\alpha \theta \\
\sigma_{\psi \psi}=\left(\beta^{2}-2\right) \frac{\partial u}{\partial r}+\beta^{2} \frac{u}{r}-\alpha \theta \\
\sigma_{z z}=\left(\beta^{2}-2\right) e-\alpha \theta
\end{gathered}
$$

where, $c_{o}=\sqrt{\frac{\lambda+2 \mu}{\rho}}$ is longitudinal wave speed; $\eta=\frac{\rho C_{E}}{K}$ is the thermal viscosity; $\varepsilon_{1}=\frac{\gamma}{\rho C_{E}}$ is the dimensionless mechanical coupling constant; $\alpha=\frac{\gamma T_{o}}{\mu}$ is the dimensionless thermoelastic coupling constant; and $\omega=a c_{o}^{2} \eta^{2}$ is the dimensionless two-temperature parameter, $\beta=\left(\frac{\lambda+2 \mu}{\mu}\right)^{1 / 2}, b=\frac{\alpha}{\beta^{2}}$ and $\varepsilon_{2}=\frac{I_{1} \delta}{C_{E} T_{o} c_{o}^{2} \eta}$. 


\subsection{The Solution in the Laplace Transform Domain}

We use the Laplace transform of both sides of the last equations defined as:

$$
\bar{f}(s)=\int_{0}^{\infty} f(t) e^{-s r} d t
$$

Hence, we obtain

$$
\begin{gathered}
\nabla^{2} \bar{e}=s^{2} \bar{e}+b \nabla^{2} \bar{\theta} ; \\
\nabla^{2} \bar{\varphi}=h \bar{\theta}+\varepsilon_{1} h \bar{e}-F(s, r) ; \\
\bar{\theta}=\bar{\varphi}-\omega \nabla^{2} \bar{\varphi} ; \\
\bar{\sigma}_{r r}=\beta^{2} \bar{e}+2 \frac{\bar{u}}{r}-\alpha \bar{\theta} ; \\
\bar{\sigma}_{\psi \psi}=\left(\beta^{2}-2\right) \bar{e}+2 \frac{\bar{u}}{r}-\alpha \bar{\theta} ; \\
\bar{\sigma}_{z z}=\left(\beta^{2}-2\right) \bar{e}-\alpha \bar{\theta} ; \\
\bar{e}=\frac{1}{r} \frac{\partial(r \bar{u})}{\partial r}=\frac{\bar{u}}{r}+\frac{\partial \bar{u}}{\partial r} ;
\end{gathered}
$$

where $F(s, r)=\varepsilon_{3} e^{-\delta r}, \varepsilon_{3}=\frac{\varepsilon_{2}\left(1+\tau_{o} s\right)}{(s+v)}$ and $h=\left(s+\tau_{o} s^{2}\right)$. All the state functions in Eqs. (18)-(24) have zero initial value. An over bar symbol denotes its Laplace transform, and $s$ denotes the Laplace transform parameter.

To simplify the solution of the above differential equations, we will consider the special case of $R=r$.

Thus, we have

$$
F(s, R)=\varepsilon_{3} e^{-\delta R}, \nabla^{2} F(s, R)=\nabla^{4} F(s, R)=0 ;
$$

By using Eqs. (19) and (20), we get

$$
\bar{\theta}=\left(1-\omega \alpha_{1}\right) \bar{\varphi}-\omega \alpha_{2} \bar{e}+\frac{\omega \alpha_{1}}{h} F(s, R) ;
$$

where $\alpha_{1}=\frac{h}{1+\omega h}$ and $\alpha_{2}=\varepsilon_{1} \alpha_{1}$.

By substituting Eq. (26) into Eqs. (18) and (19), we obtain

$$
\left(\nabla^{2}-\alpha_{1}\right) \bar{\varphi}=\alpha_{2} \bar{e}-\frac{\alpha_{1}}{h} F(s, R) ;
$$

and

$$
\left(\nabla^{2}-\alpha_{3}\right) \bar{e}=\alpha_{4} \bar{\varphi}-\frac{\alpha_{4}}{h} F(s, R)
$$

where $\alpha_{3}=\frac{s^{2}+\alpha_{2} b\left(1-\omega \alpha_{1}\right)}{1+\omega \alpha_{2} b}, \alpha_{4}=\frac{\alpha_{1} b\left(1-\omega \alpha_{1}\right)}{1+\omega \alpha_{2} b}$.

Eliminating $\bar{e}$ from Eqs. (27) and (28), we get

$$
\left[\nabla^{4}-\left(\alpha_{1}+\alpha_{3}\right) \nabla^{2}+\left(\alpha_{1} \alpha_{3}-\alpha_{2} \alpha_{4}\right)\right] \bar{\varphi}=\alpha_{5} F(s, R) ;
$$

where $\alpha_{5}=\frac{\left(\alpha_{1} \alpha_{3}-\alpha_{2} \alpha_{4}\right)}{h}$.

In a similar manner, we can show that $\bar{e}$ satisfies the equation

$$
\left[\nabla^{4}-\left(\alpha_{1}+\alpha_{3}\right) \nabla^{2}+\left(\alpha_{1} \alpha_{3}-\alpha_{2} \alpha_{4}\right)\right] \bar{e}=0 .
$$

For finite solutions, the solutions of Eqs. (28) and (29) take the form

$$
\bar{\varphi}=\frac{F\left(s, R_{0}\right)}{h}+\sum_{i=1}^{2} A_{i} K_{0}\left(p_{i} r\right)
$$

and

$$
\bar{e}=\sum_{i=1}^{2} B_{i} K_{0}\left(p_{i} r\right)
$$

where $K_{0}()$ is the modified Bessel function of the second kind of order zero. $A_{1}, A_{2}, B_{1}$, and $B_{2}$ are all parameters depending on the parameter $s$ of the Laplace transform.

$p_{1}^{2}$ and $p_{2}^{2}$ are the roots of the characteristic equation

$$
p^{4}-\left(\alpha_{1}+\alpha_{3}\right) p^{2}+\left(\alpha_{1} \alpha_{3}-\alpha_{2} \alpha_{4}\right)=0
$$

Using Eq. (29), we obtain

$$
B_{i}=\alpha_{4} A_{i}, \quad i=1,2 .
$$

Substituting Eq. (34) into Eq. (32), we get

$$
\bar{e}=\alpha_{4} \sum_{i=1}^{2} A_{i} K_{0}\left(p_{i} r\right)
$$

Substituting Eq. (35) into Eq. (24), we obtain

$$
\bar{u}=-\alpha_{4} \sum_{i=1}^{2} \frac{A_{i}}{p_{i}} K_{1}\left(p_{i} r\right)
$$

where $K_{1}()$ is the modified Bessel function of the second kind of order one.

In deriving Eq. (36), we have used the following well-known relation of the Bessel function:

$$
\int z K_{0}(z) d z=-z K_{1}(z)
$$

Using Eqs. (31) and (35) in Eq. (26), we obtain

$$
\bar{\theta}=\frac{F\left(s, R_{0}\right)}{h}+\sum_{i=1}^{2} \theta_{i} A_{i} K_{0}\left(p_{i} r\right)
$$

where

$$
\theta_{i}=\left(1-\omega \alpha_{1}\right)\left(p_{i}^{2}-\alpha_{3}\right)-\omega \alpha_{2} \alpha_{4} \quad i=1,2 .
$$

Finally, substituting Eqs. (35), (36), and (37) into Eqs. (21)(23), we obtain the stress components in the form

$$
\begin{aligned}
& \bar{\sigma}_{r r}=-\frac{\alpha F(s, R)}{h} \\
& +\sum_{i=1}^{2} A_{i}\left[\left(\beta^{2} \alpha_{4}-\alpha \theta_{i}\right) K_{0}\left(p_{i} r\right)+\frac{2 \alpha_{4}}{r p_{i}} K_{1}\left(p_{i} r\right)\right]
\end{aligned}
$$

$$
\begin{aligned}
& \bar{\sigma}_{\psi \psi}=-\frac{\alpha F(s, R)}{h} \\
&+\sum_{i=1}^{2} A_{i}\left[\left(\left(\beta^{2}-2\right) \alpha_{4}-\alpha \theta_{i}\right) K_{0}\left(p_{i} r\right)-\frac{2 \alpha_{2}}{r p_{i}} K_{1}\left(p_{i} r\right)\right]
\end{aligned}
$$

$$
\sigma_{z z}=-\frac{\alpha F(s, R)}{h}+\sum_{i=1}^{2}\left[\left(\beta^{2}-2\right) \alpha_{4}-\alpha \theta_{i}\right] A_{i} K_{0}\left(p_{i} r\right)
$$


To complete the solution in the Laplace transform space, we will consider the medium described above as quiescent and the bounding plane of the cavity $(r=R)$ traction free, with no thermal loading accept the laser beam:

$$
\varphi(R, t)=0 .
$$

After using Laplace transform, we have

$$
\bar{\varphi}(R, s)=0 .
$$

Also, we have

$$
\sigma_{r r}(R, t)=0 .
$$

After using Laplace transform, we get

$$
\bar{\sigma}_{r r}(R, s)=0 \text {. }
$$

Applying the last two conditions gives

$$
\sum_{i=1}^{2} A_{i}\left(p_{i}^{2}-\alpha_{3}\right) K_{0}\left(p_{i} R\right)=-\frac{F(s, R)}{h} ;
$$

and

$$
\begin{array}{r}
\sum_{i=1}^{2} A_{i}\left[\left(\beta^{2} \alpha_{4}-\alpha \theta_{i}\right) K_{0}\left(p_{i} R\right)+\frac{2 \alpha_{4}}{R p_{i}} K_{1}\left(p_{i} R\right)\right] \\
=\frac{\alpha F(s, R)}{h} .
\end{array}
$$

Solving the last system of equations gives

$$
\left[\begin{array}{l}
A_{1} \\
A_{2}
\end{array}\right]=\left[\begin{array}{ll}
l_{11} & l_{12} \\
l_{21} & l_{22}
\end{array}\right]^{-1}\left[\begin{array}{c}
-1 \\
\alpha
\end{array}\right] \frac{F(s, R)}{h}
$$

hence $A_{1}=\frac{F(s, R)\left(\alpha l_{12}-l_{22}\right)}{h\left(l_{11} l_{22}-l_{12} l_{21}\right)}$ and $A_{2}=\frac{F(s, R)\left(l_{21}-\alpha l_{11}\right)}{h\left(l_{11} l_{22}-l_{12} l_{21}\right)}$, where

$$
\begin{gathered}
l_{11}=\left(p_{1}^{2}-\alpha_{3}\right) K_{0}\left(p_{1} r\right) \\
l_{12}=\left(p_{2}^{2}-\alpha_{3}\right) K_{0}\left(p_{2} r\right) ; \\
l_{21}=\left(\beta^{2} \alpha_{4}-\alpha \theta_{1}\right) K_{0}\left(p_{1} R\right)+\frac{2 \alpha_{4}}{R p_{1}} K_{1}\left(p_{1} R\right) ;
\end{gathered}
$$

and

$$
l_{22}=\left(\beta^{2} \alpha_{4}-\alpha \theta_{2}\right) K_{0}\left(p_{2} R\right)+\frac{2 \alpha_{4}}{R p_{2}} K_{1}\left(p_{2} R\right) .
$$

Finally, we obtain the solutions in the Laplace transform domain as in Eqs. (48)-(52) (top of the next page).

\section{NUMERICAL INVERSION OF LAPLACE TRANSFORM}

In order to determine the conductive and thermal temperature, displacement, and stress distributions in the time domain, the Riemann-sum approximation method is used to obtain the numerical results. In this method, any function in Laplace domain can be inverted to the time domain as

$$
f(t)=\frac{e^{\kappa t}}{t}\left[\frac{1}{2} \bar{f}(\kappa)+R e \sum_{n=1}^{N}(-1)^{n} \bar{f}\left(\kappa+\frac{i n \pi}{t}\right)\right]
$$

where $R e$ is the real part and $i$ is imaginary number unit. For faster convergence, multiple numerical experiments have shown that the value of $\kappa$ satisfies the relation $\kappa t \approx 4.7 .^{8}$

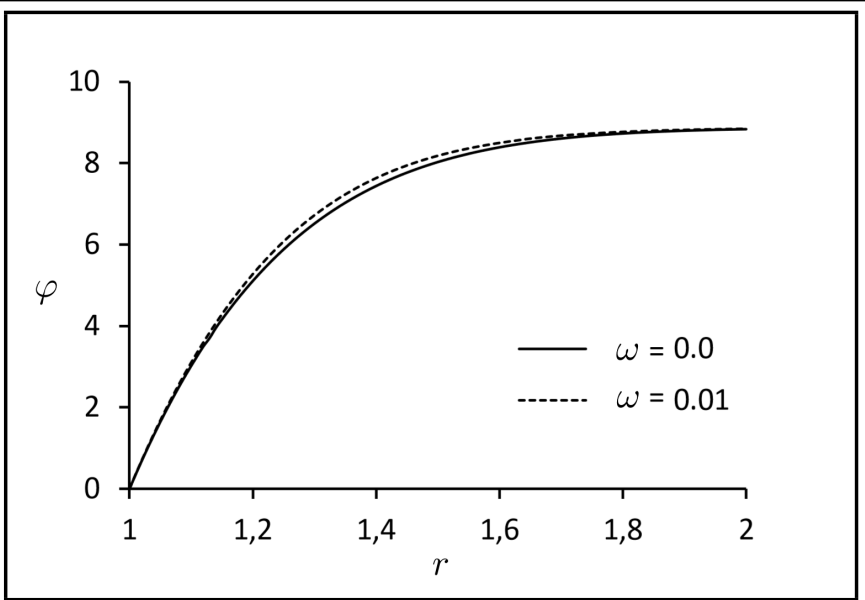

Figure 1. The conductive temperature with different value two-temperature parameter.

\subsection{Numerical Results and Discussion}

With a view to illustrating the analytical procedure presented earlier, we now consider a numerical example for which computational results are given. For this purpose, copper is taken as the thermoelastic material for which we take the following values of the different physical constants: ${ }^{.25}$

$$
\begin{aligned}
K & =386 \mathrm{~kg} \mathrm{~m} \mathrm{~K}^{-1} \mathrm{~s}^{-3} & \alpha_{T} & =1.78(10)^{-5} \mathrm{~K}^{-1} ; \\
\rho & =8954 \mathrm{~kg} \mathrm{~m}^{-3} ; & C_{E} & =383.1 \mathrm{~m}^{2} \mathrm{~K}^{-1} \mathrm{~s}^{-2} ; \\
T_{o} & =293 \mathrm{~K} ; & \mu & =3.86(10)^{10} \mathrm{~kg} \mathrm{~m}^{-1} \mathrm{~s}^{-2} ; \\
\lambda & =7.76(10)^{10} \mathrm{~kg} \mathrm{~m}^{-1} \mathrm{~s}^{-2} ; & \beta^{2} & =4 ; \\
R & =1.0 ; & \tau_{o} & =0.02 ; \\
t & =0.1 . & &
\end{aligned}
$$

From the above values, we get the non-dimensional values of the problem as:

$$
b=0.01041, \quad \alpha=0.0417232, \quad \varepsilon_{1}=1.618, \quad \varepsilon_{2}=10^{2} .
$$

Figures 1-5 represent the distributions of the conductive temperature, the dynamic-temperature, the stress, the displacement and the strain respectively when $v=0.1, \delta=0.1$ and with different value of two-temperature parameter $\omega=$ $0.0,0.01$ to stand on the effect of this parameter on all the studied filed. This group of figures shows that, the two-temperature parameter has significant effects on all the state of functions of the thermoelastic materials. The two-temperature parameter makes the sharp points in the stress, the strain and the displacement distribution disappeared.

Figures 6-10 represent the distributions of the conductive temperature, the thermo-dynamic temperature, the stress, the displacement and the strain respectively when $\omega=0.01, v=$ 0.1 and with different value of absorption coefficient parameter $\delta=0.1,0.05$ to stand on the effect of this parameter on all the studied filed. This group of figures shows that, the absorption coefficient parameter has significant effects on all the states of functions of the thermoelastic materials. When the value of the absorption coefficient parameter increases, all the state functions of the material decrease.

Figures 11-15 represent the distributions of the conductive temperature, thermo-dynamic temperature, stress, displacement, and strain, respectively, when $\omega=0.01, \delta=0.1$ and 


$$
\begin{aligned}
& \bar{\varphi}=\frac{F(s, R)}{h}\left[1+\frac{1}{\left(l_{11} l_{22}-l_{12} l_{21}\right)}\left[\left(\alpha l_{12}-l_{22}\right)\left(p_{1}^{2}-\alpha_{3}\right) K_{0}\left(p_{1} r\right)+\left(l_{21}-\alpha l_{11}\right)\left(p_{2}^{2}-\alpha_{3}\right) K_{0}\left(p_{2} r\right)\right]\right] ; \\
& \bar{\theta}=\frac{F(s, R)}{h\left(l_{11} l_{22}-l_{12} l_{21}\right)} \\
& {\left[1+\left(\left(1-\omega \alpha_{1}\right)\left(p_{1}^{2}-\alpha_{3}\right)-\omega \alpha_{2} \alpha_{4}\right)\left(\alpha l_{12}-l_{22}\right) K_{0}\left(p_{1} r\right)+\left(\left(1-\omega \alpha_{1}\right)\left(p_{2}^{2}-\alpha_{3}\right)-\omega \alpha_{2} \alpha_{4}\right)\left(l_{21}-\alpha l_{11}\right) K_{0}\left(p_{2} r\right)\right] \text {; }} \\
& \bar{\sigma}_{r r}=\frac{F(s, R)}{h}\left[-\alpha+\frac{\left(\alpha l_{12}-l_{22}\right)}{\left(l_{11} l_{22}-l_{12} l_{21}\right)}\left[\left(\beta^{2} \alpha_{4}-\alpha \theta_{1}\right) K_{0}\left(p_{1} r\right)+\frac{2 \alpha_{4}}{r p_{1}} K_{1}\left(p_{1} r\right)\right]\right. \\
& \left.+\frac{\left(l_{21}-\alpha l_{11}\right)}{\left(l_{11} l_{22}-l_{12} l_{21}\right)}\left[\left(\beta^{2} \alpha_{4}-\alpha \theta_{2}\right) K_{0}\left(p_{2} r\right)+\frac{2 \alpha_{4}}{r p_{2}} K_{1}\left(p_{2} r\right)\right]\right] ; \\
& \bar{e}=\frac{\alpha_{4} F(s, R)}{h\left(l_{11} l_{22}-l_{12} l_{21}\right)}\left[\left(\alpha l_{12}-l_{22}\right) K_{0}\left(p_{1} r\right)+\left(l_{21}-\alpha l_{11}\right) K_{0}\left(p_{2} r\right)\right] ; \\
& \bar{u}=-\frac{\alpha_{4} F(s, R)}{h p_{1} p_{2}\left(l_{11} l_{22}-l_{12} l_{21}\right)}\left[p_{2}\left(\alpha l_{12}-l_{22}\right) K_{1}\left(p_{1} r\right)+p_{1}\left(l_{21}-\alpha l_{11}\right) K_{1}\left(p_{2} r\right)\right]
\end{aligned}
$$

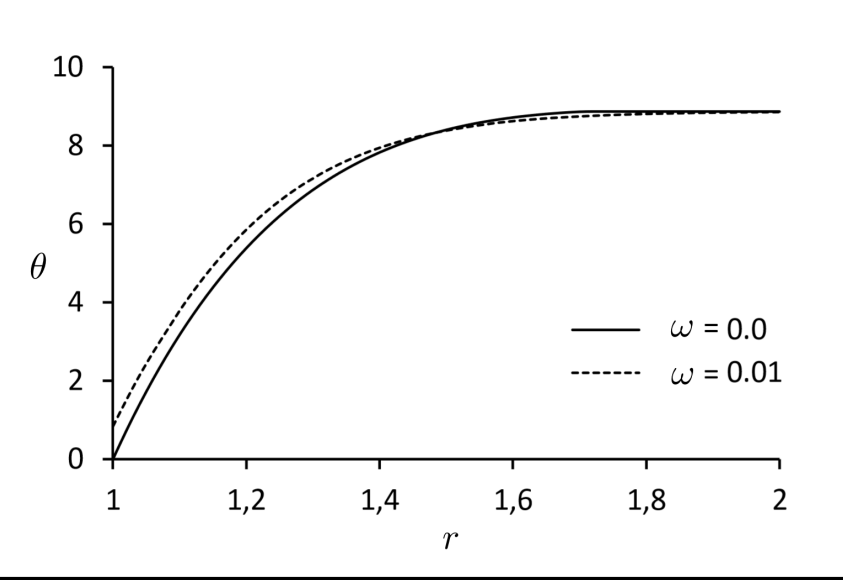

Figure 2. The thermo-dynamic temperature with different value twotemperature parameter.

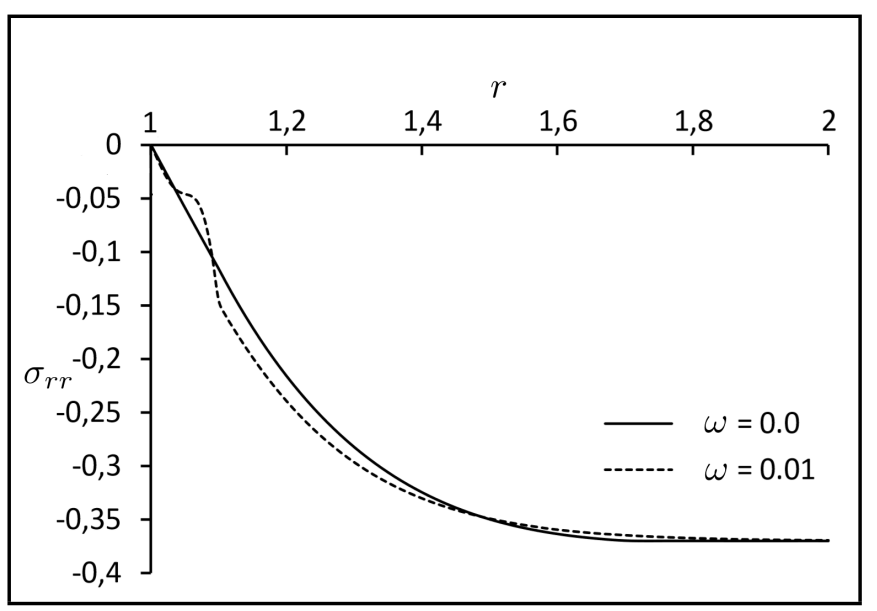

Figure 3. The stress with different value two-temperature parameter.

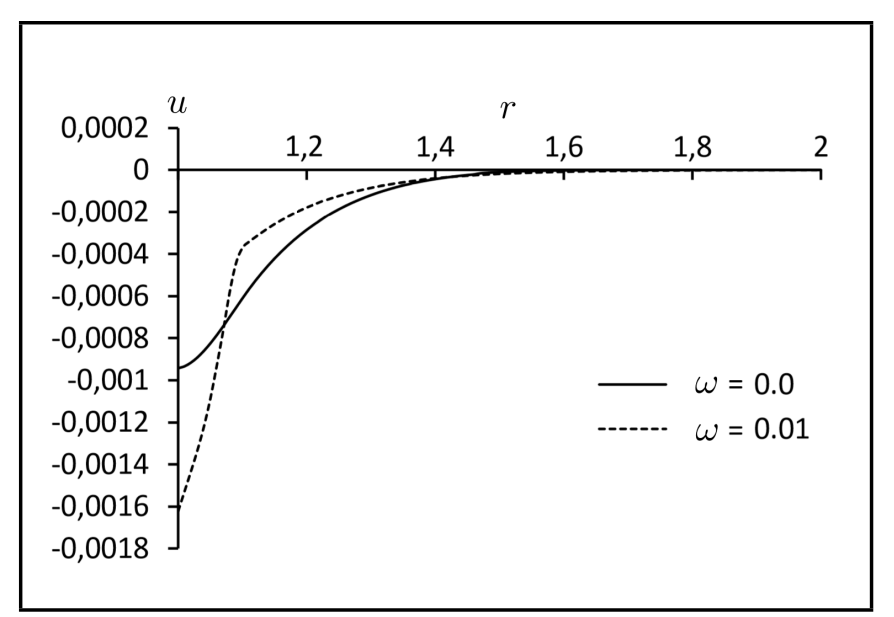

Figure 4. The displacement with different value two-temperature parameter.

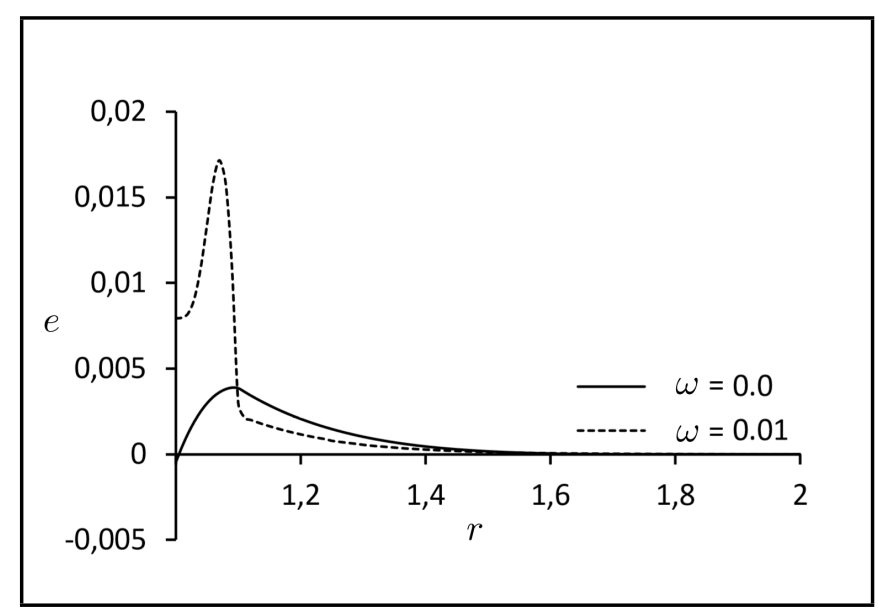

Figure 5. The strain with different value two-temperature parameter. 


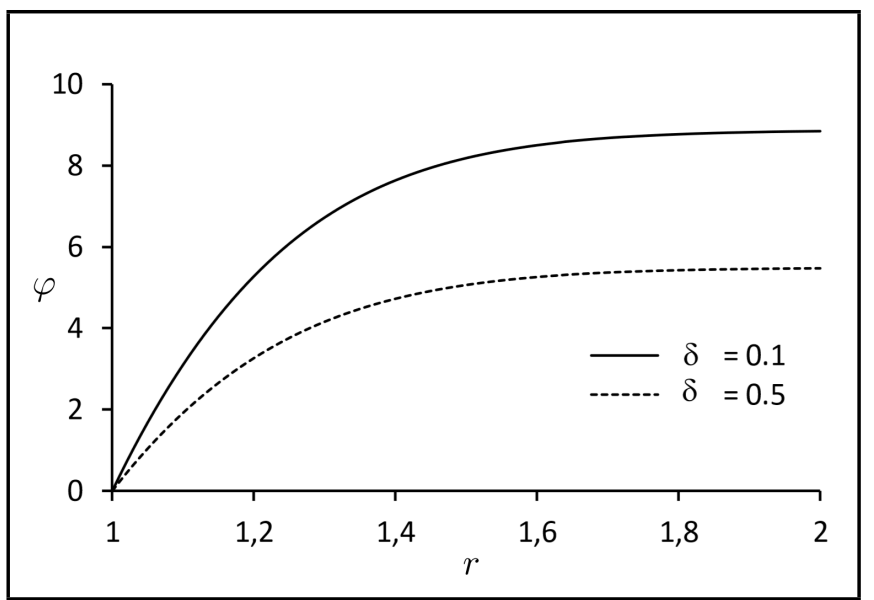

Figure 6. The conductive temperature with different value of absorption coefficient.

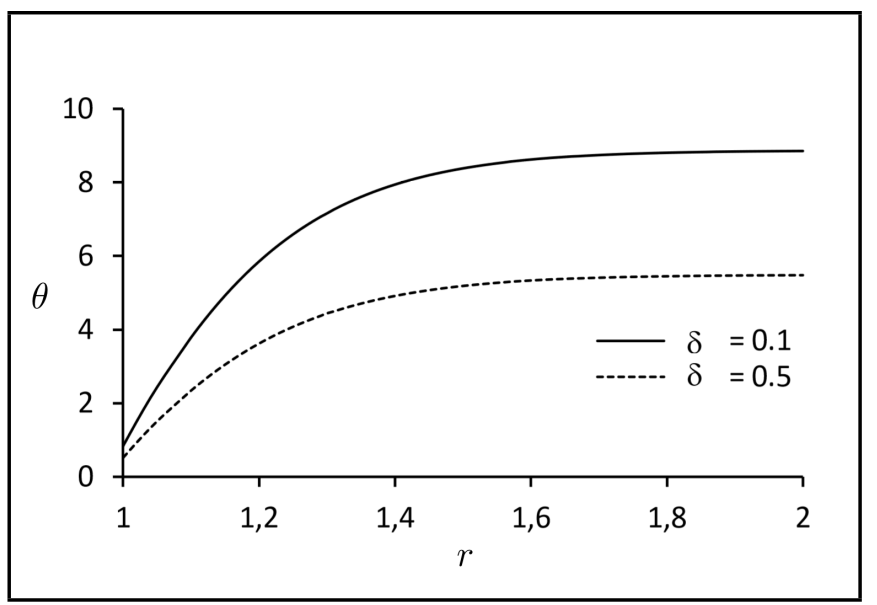

Figure 7. The thermo-dynamic temperature with different value of absorption coefficient.

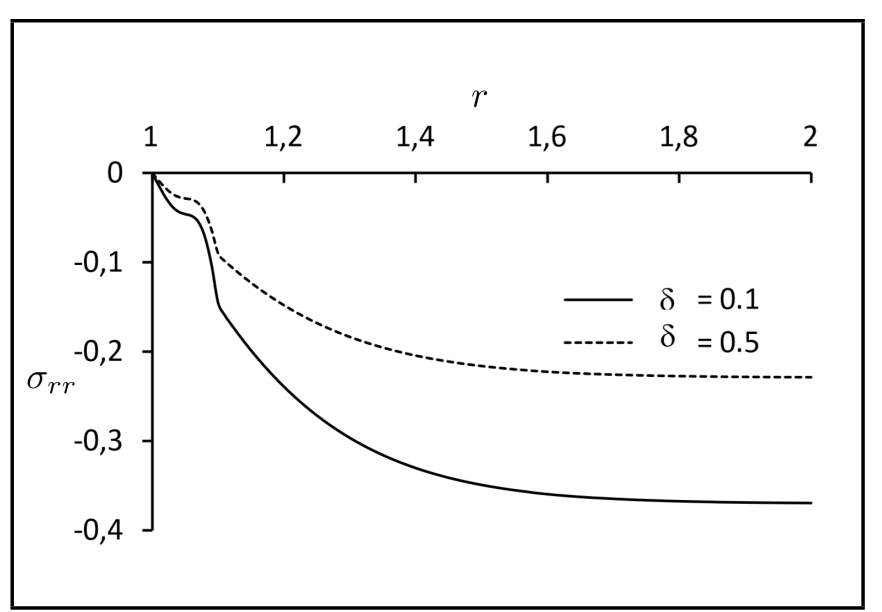

Figure 8. The stress with different value of absorption coefficient parameter.

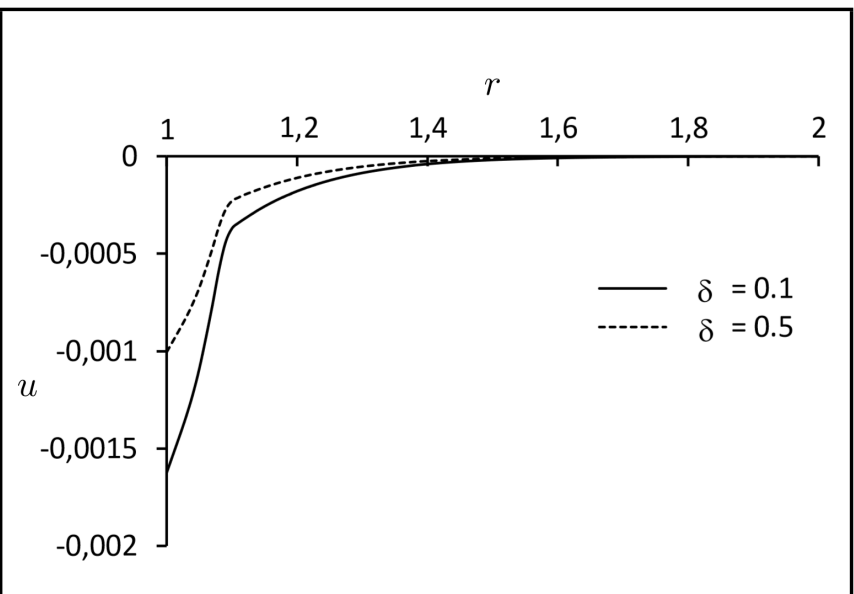

Figure 9. The displacement with different value of absorption coefficient.

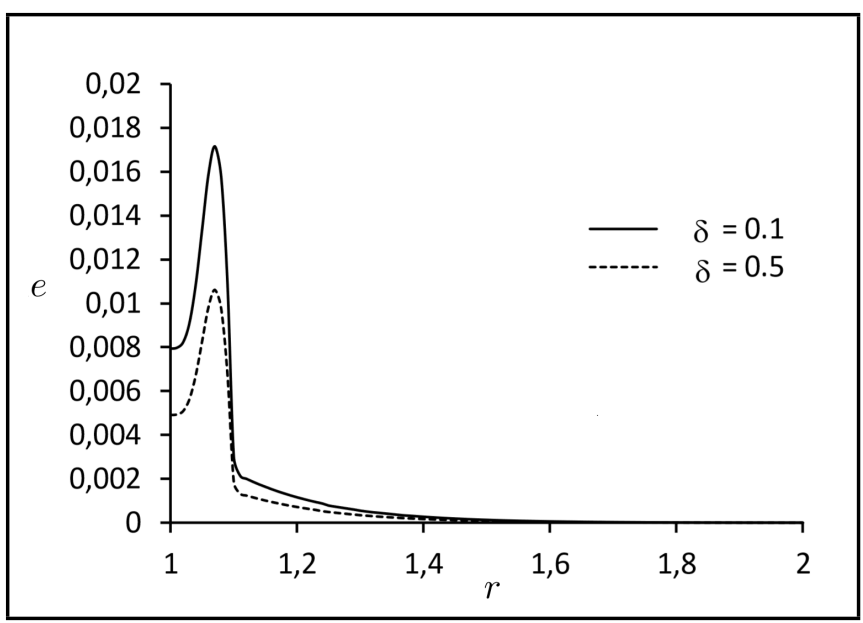

Figure 10. The strain with different value of absorption coefficient.

with different value of laser pulse parameter $v=0.1,0.05$ to stand on the effect of this parameter on all the studied filed. This group of figures shows that the laser pulse parameter has significant effects on the distributions of the conductive temperature, the thermo-dynamic temperature and the stress, while it has weak effects on the distribution of the displacement and the strain. When the value of laser pulse parameter increases, all the state functions of the material decrease.

\section{CONCLUSION}

In this work, a studying of the induced temperature and stress fields in an elastic infinite medium with cylindrical cavity under the purview of two-temperature thermoelasticity has been done. The medium has been considered to be an isotropic homogeneous thermoelastic material. The bounding plane surface of the cavity is loaded thermally by time exponentially decaying laser pulse and we found the following:

1. The two-temperature parameter has a significant effect on all the studied fields.

2. The absorption coefficient parameter has a significant effect on all the studied fields.

3. When the value of the absorption coefficient parameter increases, all the state functions of the material decrease. 


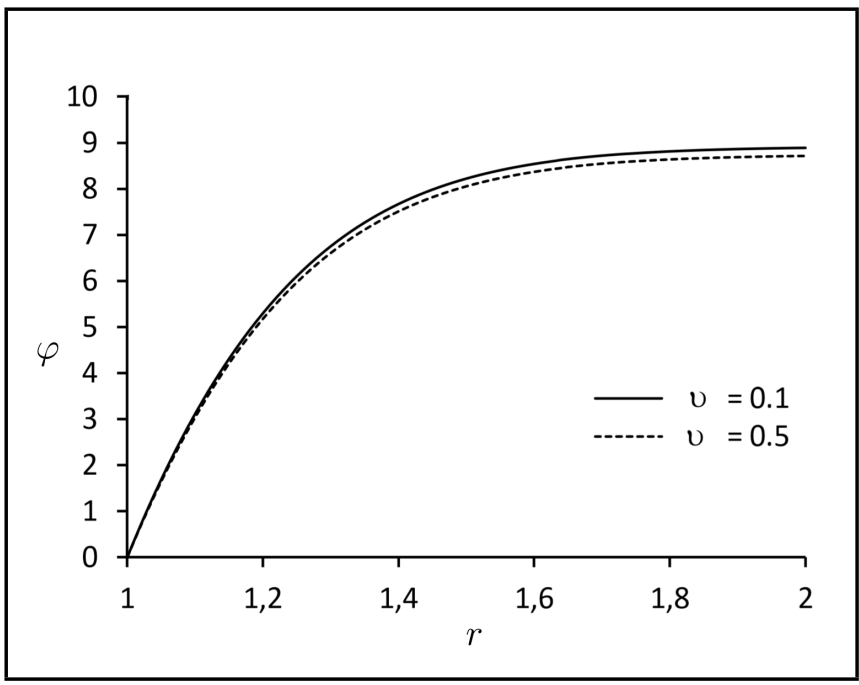

Figure 11. The conductive temperature with different value of laser pulse parameter.

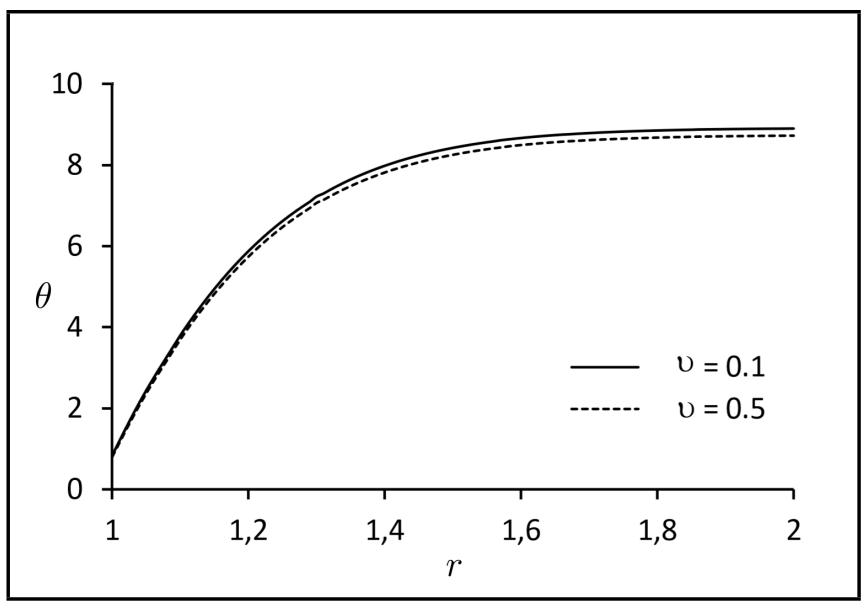

Figure 12. The thermo-dynamic temperature with different value of laser pulse parameter.

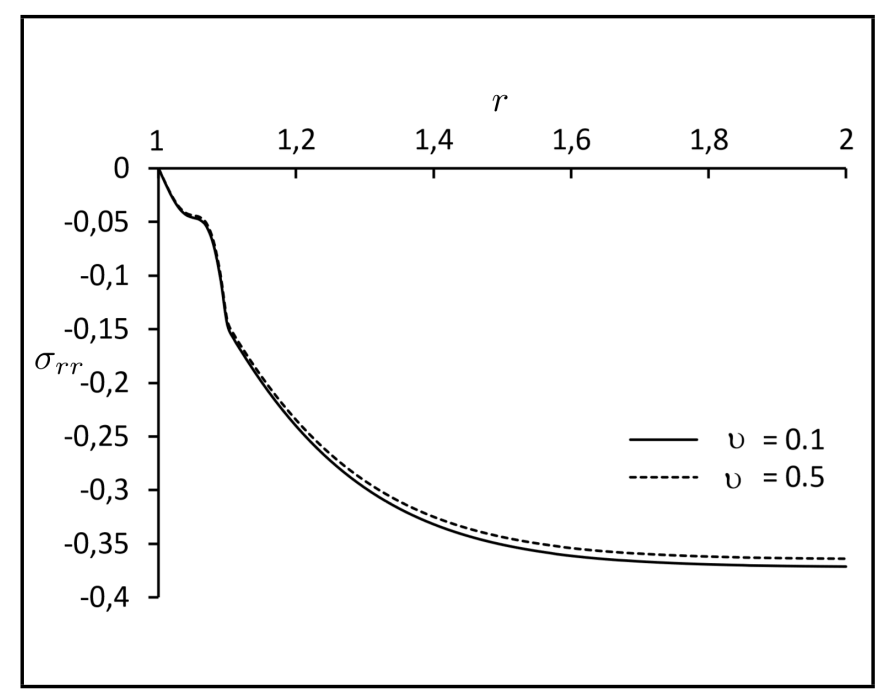

Figure 13. The stress with different value of laser pulse parameter.

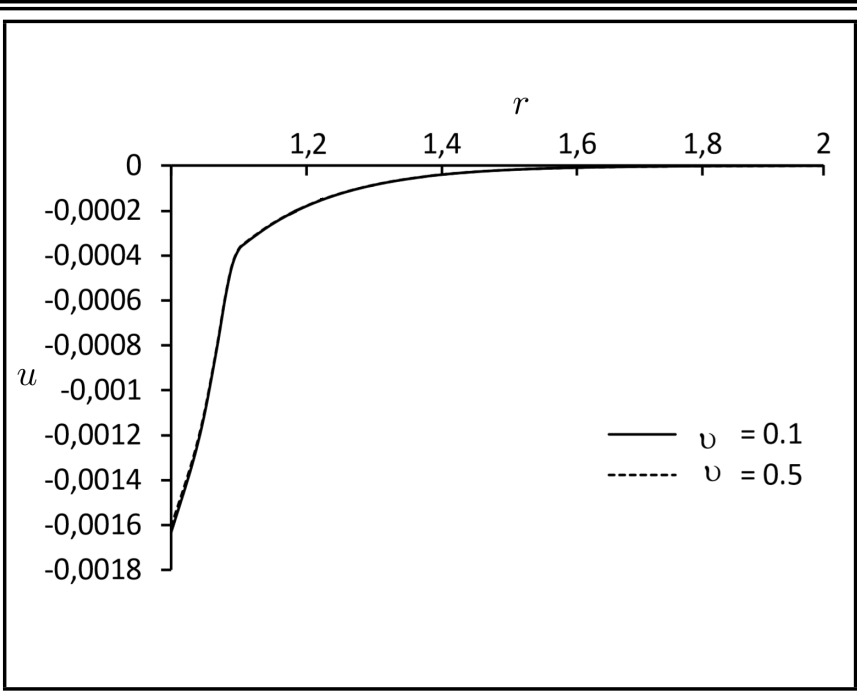

Figure 14. The displacement with different value of laser pulse parameter.

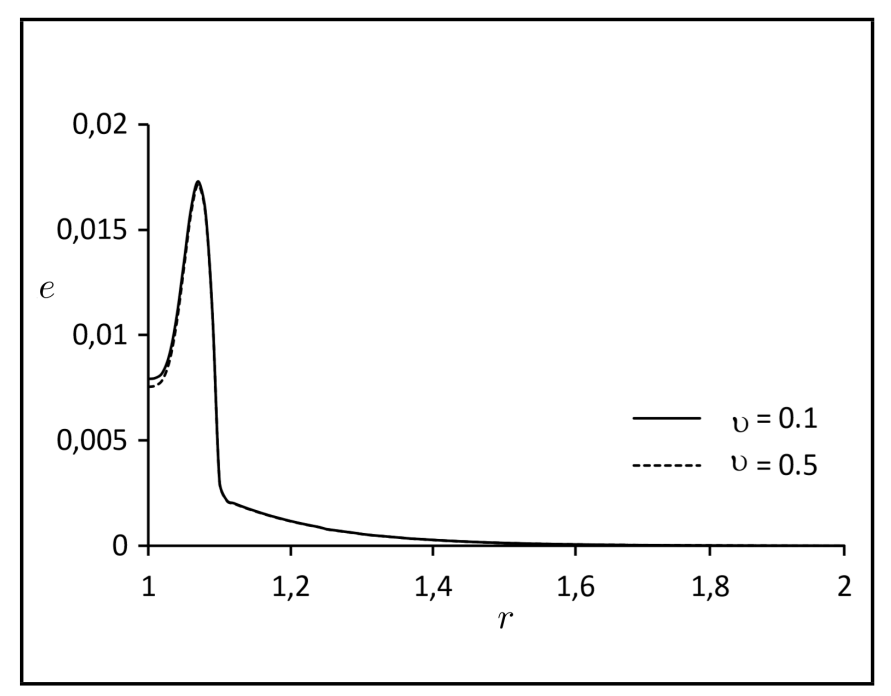

Figure 15. The strain with different value of laser pulse parameter.

4. The laser pulse parameter has significant effects on the distributions of the conductive temperature, the thermodynamic temperature, and the stress, while it has weak effects on the distribution of the displacement and the strain.

5. When the value of laser pulse parameter increases, all the state functions of the material decrease.

\section{REFERENCES}

1 Biot, M. A. Thermoelasticity and irreversible thermodynamics, Journal of Applied Physics 27(3), 240-253 (1956). http://dx.doi.org/10.1063/1.1722351

2 Bargmann, H. Recent developments in the field of thermally induced waves and vibrations, $\mathrm{Nu}$ clear Engineering and Design 27(3), 372-385 (1974). http://dx.doi.org/10.1016/0029-5493(74)90181-2

3 Boley, B. A. Thermal stresses: a survey, Springer, (1980). http://dx.doi.org/10.1007/978-1-4613-3156-8_1

${ }^{4}$ Chandrasekharaiah, D. S. Thermoelasticity with second sound: a review, Applied Mechanics Reviews 39(3), 355376 (1986). http://dx.doi.org/10.1115/1.3143705 
${ }^{5}$ Lord, H. W., Shulman, Y. A generalized dynamical theory of thermoelasticity, Journal of the Mechanics and Physics of Solids 15(5), 299-309 (1967). http://dx.doi.org/10.1016/0022-5096(67)90024-5

${ }^{6}$ Green, A. E., Lindsay, K. A. Thermoelasticity, Journal of Elasticity 2(1), 1-7 (1972). http://dx.doi.org/10.1007/BF00045689

${ }^{7}$ Sun, Y., Fang, D., Saka, M., Soh, A. K. Laserinduced vibrations of micro-beams under different boundary conditions, International Journal of Solids and Structures 45(7), 1993-2013 (2008). http://dx.doi.org/10.1016/j.jissolstr.2007.11.006

8 Tzou, D. Y. Macro-to micro-scale heat transfer: the lagging behavior, CRC Press, (1996). ISBN-10: 156032435X

9 Tzou, D. Y. Macro-to microscale heat transfer: The lagging behavior, In. Taylor \& Francis (Washington, DC), (1997).

10 Wang, X., Xu, X. Thermoelastic wave induced by pulsed laser heating, Applied Physics A 73(1), 107-114 (2001). http://dx.doi.org/10.1007/s003390000593

11 Gurtin, M. E., Williams, W. O. An axiomatic foundation for continuum thermodynamics, Archive for Rational Mechanics and Analysis 26(2), 83-117 (1967). http://dx.doi.org/10.1007/BF00285676

${ }^{12}$ Chen, P. J., Gurtin, M. E. On a theory of heat conduction involving two temperatures, Zeitschrift fr Angewandte Mathematik und Physik ZAMP 19(4), 614-627 (1968). http://dx.doi.org/10.1007/BF01594969

${ }^{13}$ Chen, P. J., Gurtin, M. E., Williams, W. O. On the thermodynamics of non-simple elastic materials with two temperatures, Zeitschrift fr angewandte Mathematik und Physik ZAMP 20(1), 107-112 (1969). http://dx.doi.org/10.1007/BF01591120

${ }^{14}$ Chen, P. J., Williams, W. O. A note on nonsimple heat conduction, Zeitschrift fr angewandte Mathematik und Physik ZAMP 19(6), 969-970 (1968). http://dx.doi.org/10.1007/BF01602278

15 Ieşan, D. On the linear coupled thermoelasticity with two temperatures, Zeitschrift fr Angewandte Mathematik und Physik ZAMP 21(4), 583-591 (1970). http://dx.doi.org/10.1007/BF01587687

16 Abbas, I. A. Eigenvalue approach for an unbounded medium with a spherical cavity based upon twotemperature generalized thermoelastic theory, Journal of Mechanical Science and Technology, 28(10), 4193-4198 (2014). http://dx.doi.org/10.1007/s12206-014-0932-6
17 Abbas, I. A. A problem on functional graded material under fractional order theory of thermoelasticity, Theoretical and Applied Fracture Mechanics, 74, 18-22 (2014). http://dx.doi.org/10.1016/j.tafmec.2014.05.005

18 Abbas, I. A. A GN model based upon twotemperature generalized thermoelastic theory in an unbounded medium with a spherical cavity, Applied Mathematics and Computation, 245, 108-115 (2014). http://dx.doi.org/10.1016/j.amc.2014.07.059

19 Abbas, I. A. Fractional order GN model on thermoelastic interaction in an infinite fibre-reinforced anisotropic plate containing a circular hole, Journal of Computational and Theoretical Nanoscience, 11(2), 380-384 (2014). http://dx.doi.org/10.1166/jctn.2014.3363

20 Abbas, I. A. Three phase lag model on thermoelastic interaction in an unbounded fiber-reinforced anisotropic medium with a cylindrical cavity, Journal of Computational and Theoretical Nanoscience, 11(4), 987-992 (2014). http://dx.doi.org/10.1166/jctn.2014.3454

${ }^{21}$ Quintanilla, R. On existence, structural stability, convergence and spatial behavior in thermoelasticity with two temperatures, Acta Mechanica 168(1-2), 61-73 (2004). http://dx.doi.org/10.1007/s00707-004-0073-6

22 Youssef, H. M. Theory of two-temperaturegeneralized thermoelasticity, IMA journal of applied mathematics 71(3), 383-390 (2006). http://dx.doi.org/10.1093/imamat/hxh101

${ }^{23}$ Puri, P., Jordan, P. M. On the propagation of harmonic plane waves under the two-temperature theory, International journal of engineering science 44(17), 1113-1126 (2006). http://dx.doi.org/10.1016/j.ijengsci.2006.07.002

${ }^{24}$ Magaña, A., Quintanilla, R. Uniqueness and growth of solutions in two-temperature generalized thermoelastic theories, Mathematics and Mechanics of Solids 14(7), 622-634 (2009). http://dx.doi.org/10.1177/1081286507087653

25 Youssef, H. M. Generalized thermoelastic infinite medium with cylindrical cavity subjected to moving heat source, Mechanics Research Communications 36(4), 487-496 (2009). http://dx.doi.org/10.1016/j.mechrescom.2008.12.004 